\title{
La crisis del Narcisismo en la teoría educativa. Hacia un nuevo realismo pedagógico.
}

Sara Morgenstern de Finkel

\section{Introducción}

A principios de los años 60 , las disciplinas relacionadas con la educación cobraron una importancia hasta entonces desconocida. Además de adquirir un status de respetabilidad académica, se les reconoció importancia en el campo de la planificación económica-social. Los nuevos aires provenían de una nueva ideología, encarnada en la Alianza para el Progreso patrocinada por la Administración Kennedy, cuyo punto de partida era la convicción de que el atraso y la miseria eran superables dentro del marco capitalista.

En tanto las reformas debían plantearse de forma planificada y gradual, la educación se presentaba como un instrumento clave en el diseno y puesta en práctica del proyecto. El clima internacional, expresado en la "coexistencia pacífica" ofrecía un marco adecuado para imaginar un mundo donde la beligerancia estaba controlada y las sociedades nacionales se pensaban como comunidades homogéneas. La planificación permitía en este contexto prevenir los conflictos potenciales y también asegurar en un futuro el bienestar individual y social.

Como es lógico, la ciencia social prevalente en estos años no parecía preocupada demasiado por las tensiones sociales; en última instancia el análisis de la conflictividad sólo distorsionaría la óptica de asepsia y objetividad que se requiere para los expertos en planificación. No es de extranar entonces que la comunidad científica, al menos en los países centrales compartiera extensamente el paradigma neo-positivista.

En este mundo encantado, la Sociología y la Economía de la Educación invadieron la escena académica como disciplinas serias, que sustituían con un ethos modernizante a las especulaciones de los filósofos y pedagogos de viejo cuño. En España este ethos llegó tardíamente, casi con una década de retraso de la mano del Opus Dei y en una peculiar combinación de espiritualismo y materialismo tecnocrático que poco tenía que ver con las críticas que el proyecto desarrollista suscitaba ya para entonces.

La nueva ideología presentaba a la educación de forma omnipotente. Para decirlo de forma gráfica y apelando a la metáfora psicoanalítica, imperaba entre los teóricos de la educación una imagen narcisista de su propia práctica, por otra parte, desentendida de los contextos históricos reales donde se aplicaba. 
La eficacia de la educación estaba garantizada de antemano, eficacia tanto para socializar en la perpetuación del orden social, como para seleccionar a los más aptos para desempefiar los distintos roles que la sociedad adjudicaba en virtud de las necesidades del desarrollo económico. Así, el funcionalismo sociológico se unía a la Economía Marginalista de la educación en un matrimonio muy bien avenido, complementario en la división de tareas: mientras el primero informaba teóricamente de las bondades y posibilidades de la educación, la segunda se ocupaba de las cuestiones prácticas que llevarían a la planificación de los recursos humanos.

\section{La socialización y selección meritocrática}

En verdad, socialización y selección son dos problemáticas recurrentes y presentes en todas las teorías sociológicas de la educación pero lo que es peculiar del Funcionalismo es que ambos procesos transcurren en un mundo sin resistencias ni conflictos. Así como la sociedad no presenta fisuras en su interior ya que siempre el conflicto es acotado y manejable, los individuos internalizan las normas y valores que se aceptan vía consenso.

El representante más conspicuo de esta postura fue sin duda el sociólogo norteamericano Talcott Parsons. Aunque en su vasta obra el tema educativo no ocupa un lugar preferente, los escritos específicos en "El sistema de las Sociedades Modernas" y "La clase escolar como un sistema social"', refiejan acabadamente su pensamiento general. En la primera obra da cuenta acabada de la importancia adjudicada a la educación, donde insiste que estamos viviendo una profunda transformación social, producto de una revolución educativa, tan importante como las revoluciones democrática e industrial del Siglo XIX y XX. Este cambio ha aparejado la reducción de la importancia del mercado y de la organización burocrática como mecanismo de selección ocupacional. En este contexto, es casi imposible repetir el mito del millonario que se hace a sí mismo, sin ninguna cualificación educativa.

La revolución educativa supone una extensión enorme del principio de igualdad de oportunidades iniciales para todos los ciudadanos, pero que no resultan necesariamente en igualdad de desempeño, puesto que hay diferencias de habilidades o de motivación familiar e individual que incentivan de forma distinta el esfuerzo y dedicación a la tarea escolar. De este modo, las cualificaciones educativas que reflejan el desempeño de los individuos introducen elementos de diferenciación y desigualdad que a su vez determinan distintas posibilidades de acceso a los empleos.

Pero, entiéndase bien, que para Parsons esta desigualdad no implica una suerte de injusticia puesto que todos tienen las mismas oportunidades iniciales. Tampoco tiene una carga conflictiva puesto que las normas del juego son aceptables.

Estos temas los retoma Parsons en el ensayo citado "La clase escolar ..." donde afirma que las nuevas formas de desigualdad introducidas por la educación se con-

(1) PARSONS, T.: The system of modern societies. Prentice Hall, Englewood Cliffs, 1971. "The school class as a social system", en Halsey, A. H., Floud, J., anda Anderson, (eds). Education, Economy and Society. Free Press, New York, 1981. (Traduc. castallana en Gras, A. Sociolog la de la educación. Narcea, Madrid). 
trapesan por la función socializadora de la misma. En efecto, uno de sus principales efectos es legitimar las diferencias salariales y de status en tanto son resultado del desempeffo escolar. En última instancia, la socialización se desenvuelve en torno a los valores comunes de igualdad de oportunidades y logros diferenciales que son parte constitutiva de la sociedad moderna, los que aseguran la estabilidad del "consenso moral".

En Europa, la teoría de una sociedad tecnológica, que requería crecientes niveles de extensión educativa se desarrolló con otro carácter, principalmente en Inglaterra, donde la Sociología de la Educación tuvo un fuerte impacto en las políticas laboristas llevadas a cabo a través del Estado Benefactor. En principio se pensaba que las desigualdades educativas no sólo mostraban la faz injusta de la estratificación social, sino que refiejaban también una total ineficacia en el aprovechamiento de los talentos potenciales. Así, era preciso compatibilizar las oportunidades con las habilidades de modo que la educación se convirtiese realmente en la gran avenida de la movilidad social $^{2}$.

Con otros matices políticos, pero sobre la misma base del supuesto papel modernizador y nivelador de la educación, los organismos internacionales de la educación, entre ellos la UNESCO y la OECD, desarrollaron la puesta en práctica de la teoría. Y efectivamente, puede decirse que nunca en la historia de la humanidad hubo una expansión escolar tan acelerada en tan poco tiempo: entre 1960 y 1976 la matrícula mundial en los tres niveles de enseñanza se incrementó en un $77 \%$, pasando de 328 a 581 millones ${ }^{3}$.

Sin embargo, las bondades adjudicadas a la expansión educativa no iban camino de ser demostradas en la práctica. A finales ya de la década del 60 , los movimientos contestatarios de los individuos más educados en los países más desarrollados ponían en tela de juicio la capacidad socializadora de las instituciones universitarias. Para colmo, en casos como Francia e Italia, los estudiantes cuestionaban el lugar privilegiado que dentro de la estratificación social se les adjudicaba; en otras palabras, se negaban a ser los delfines de la cultura.

Desde la perspectiva actual cabe preguntarse que si los estudiantes vinculaban sus luchas a las del movimiento obrero, no sería porque intuían que el contacto con el futuro mundo laboral acortaba las distancias entre la explotación del trabajo intelectual y la explotación del conjunto de los asalariados.

Para finales de la década había un excepticismo bastante extendido acerca de la influencia de la educación en la movilidad social. Si tuvo alguna vez este papel, fue en contadas situaciones históricas, generalmente en sociedades muy abiertas y con un alto grado de expansión económica y fundamentalmente en países receptores de inmigración como los Estados Unidos, Australia o Argentina a comienzos de siglo. Este escepticismo no provenía de los sectores académicos tradicionalmente contestatarios o de izquierdas, sino también de los propios teóricos de la escuela Funcionalista.

(2) KARABEL, J., HALSEY, A.H. Power and ideology in education. Oxford University Press, 1978, pp. 1011.

(3) CARCELES, G. "Development of education in the world" International Review of Education. Vol. XXV, n. 2-3, 1979, p. 151. 
Raymond Boudon, heredero de lo mejor de la tradición durkheimiana, quien ha estudiado comparativamente el impacto de la extensión del acceso a la educación sobre la estratificación social en diversos países europeos concluye: ${ }^{4}$

"... una reducción de la desigualdad de oportunidades escolares no entraña en absoluto una atenuación de le herencia social. Lo cual no quiere decir, sin embargo, que la reducción de las desigualdades escolares no tenga necesariamente ningún efecto en el nivel de las desigualdades sociales. Lo que ocurre, sencillamente, es que los parámetros característicos de las sociedades industriales no han permitido que la reducción de las desigualdades escolares fuese acompañada por una disminución de las desigualdades sociales en el curso de los últimos decenios. Es evidente que en función de las configuraciones de los factores simultáneamente responsables de la herencia social, una reducción de la desigualdad de oportunidades escolares puede ir acompafiada por un aumento, por una disminución o por una estabilidad de la herencia social".

En una escala mucho más pequefia que los estudios de Boudon, pero que en extremo demuestra la relevancia de la socialización familiar, me gustaría referirme brevemente a la investigación de Baller y otros ${ }^{5}$. Se trata de un estudio longitudinal de 109 niños retrasados, con un coeficiente intelectual próximo a 60 , que consitió en un seguimiento hasta los 50 años. En esa edad algunos pocos estaban en instituciones especializadas, la mayoría trabajaba como obreros no cualificados o semicualificados. Pero lo realmente interesante es que un $18 \%$ tenía empleos de "cuello blanco", tales como empleado de oficina, comercio o laboratorio, policía, capataz, vendedor de propiedades, fotógrafo, comerciantes, etc. Obviamente para este último grupo, el éxito ocupacional no estaba en absoluto vinculado al nivel intelectual, más bien se demostró que los más exitosos eran aquellos cuyo comportamiento o forma de hablar y vestir eran propios de la clase media.

\section{La educación y el desarrollo económico}

El papel promotor de la educación como canal de movilidad implica entre otras cosas un ajuste funcional entre la producción educativa y la demanda de empleo; esto es, que la pirámide educativa se corresponda de la forma más aproximada posible a la pirámide ocupacional. Operativamente, ya dentro del marco de la planificación de los afios 60 , la necesidad de este ajuste significó un reconocimiento al papel dinamizador de la educación en el desarrollo económico, tal como lo condensa el concepto de capital humano.

La introducción de este concepto en la explicación del desarrollo histórico de los países hoy avanzados permitía un giro bastante importante con respecto a las formulaciones ortodoxas. En efecto, según estas teorias, el famoso despegue rostowniano no puede explicarse exclusivamente por los factores económicos clásicos (tierra-

(4) BOUDON, RAYMOND, LAGNEAU, JANDNA. "Las desigualdades de oportunidades de educación en Europa Occidental". Perspectivas. UNESCO. Vol. X n.?2, 1980, p. 205.

(5) BALLER, W.R. et al. "Midlife attainment of the mentally retarded" Genetic Psychology Monographs 42, 1967, citado en Collins, R. The credential society, Academic Press, 1979, Pp. 28-29. 
capital-trabajo). El plus de productividad marginal, no cuantificable por los factores anteriores, sólo es atribuible a la formación técnica de la fuerza de trabajo. Dentro de este razonamiento, la Economía Marginalista de la Educación ha considerado a la educación como un factor residual importante y decisivo que explicarían los aspectos no suceptibles de cuantificar en el origen de la Revolución Industrial.

Las evidencias históricas cuestionan este supuesto, al menos en Inglaterra donde se han hecho numerosos estudios locales. Sanderson, por ejemplo, demuestra que en Lancashire la Revolución Industrial redujo más que estimuló la educación elemental puesto que ésta era irrelevante para el trabajo en fábrica y más aún para la movilidad social.

Lo sorprendente del caso inglés es que no hubo durante el siglo XIX una política firme que impulsara la educación técnica. Tampoco hubo una presión significativa por parte de los empresarios, excepto en algunos casos excepcionales como George Birbeck $y$ otros pocos que participaron en el llamado "Movimiento pro Institutos de Mecánica". Roderick y Stephens lamentan, por las consecuencias ulteriores en el desarrollo inglés, la falta de un impulso empresario a la educación técnica. Los Institutos de Mecánica que florecieron en la era victoriana estaban más preocupados por lograr el control social sobre las clases trabajadoras que por desarrollar una educación técnicocientífica, como fue el caso de la Realschulen en Alemania ${ }^{7}$. Durante muchos años el poderío industrial británico descansó en los aficionados, el artesano-inventor, el propietario fabril, en otras palabras, en los "self-made men", hombres de la páctica y no de las universidades.

Si las evidencias históricas no dejan muy bien colocada a la educación como factor de desarrollo, el análisis actual de los procesos de trabajo tampoco refleja un ajuste significativo entre el sector educativo y el sector productivo. Para que la educación se constituya en un factor de desarrollo, son necesarias, al menos las siguientes condiciones:

1. Que exista una oferta de empleo donde se inserten los "recursos humanos producidos por el sistema educativo.

2. Que el contenido técnico de los distintos trabajos guarde una estrecha relación con las cualificaciones provistas por la educación formal.

3. Que los saberes y habilidades de la fuerza de trabajo sean utilizados y potenciados de forma que contribuyan a la innovación en la tecnología y organización de la. producción.

Estas condiciones, nunca suficientemente probadas empíricamente, consituian, por decirlo de alguna forma, la base del optimismo de los affos 60 . No sólo se daba por sentado que habría pleno empleo en los países centrales, sino que se requeririan niveles de cualificación cada vez más altos para cubrir los puestos de creciente complejidad tecnológica.

A partir de la crisis que comenzó a evidenciarse aproximadamente en 1973, se comprobaron serias limitaciones a la expansión del empleo y con perspectivas poco prometedoras para el futuro, aún dentro de los paises centrales. El Instituto Económico

(6) SANDERSON, M.: "Literacy and social mobility in England. Past and Present, n. ${ }^{2}$ 56, August 1972.

(7) RODERICK, G., STEPHENS, M.D. Education and industry in the nineteenth Century: The english desease. Longman, 1978. 
de Rotterdam preve para los países de la Comunidad Económica Europea una tasa de desempleo del 12,1\% para 1990, con una alternativa optimista basada en la hipótesis de un alto crecimiento económico de $9,4 \%$ y una alternativa pesimista del $18 \%$. En Espaffa, con más de tres millones de parados, la proporción alcanza ya al $20,77 \%$ de la fuerza laboral ${ }^{8}$.

El análisis de los procesos de trabajo muestra por otra parte, una tendencia histórica constante a la parcelación de tareas y a la separación cada vez más tajante entre la concepción y la ejecución. Desde el Taylorismo hasta la robotización actual, pasando por el Fordismo y el Neo-Fordismo, la organización del trabajo ha acentuado la pérdida del control por parte del trabajador sobre su trabajo y por tanto, una constante degradación de sus capacidades y saberes.

Braverman ${ }^{9}$ quien ha sido un autor pionero en el análisis de los procesos de trabajo en el capitalismo tardío, ha mostrado cómo esas tendencias iniciadas en la industria se han extendido a los servicios, incluída la ensenfanza ${ }^{10}$. El resultado general se expresa en una descualificación de la fuerza de trabajo que Braverman ve como un proceso general e irreversible.

Los sociólogos actuales matizan bastante las formulaciones de Braverman en un doble sentido. Por una parte no es una tendencia homogénea que afecte inevitablemente y por igual a todos los sectores de la economia, entre otras cosas depende de las resistencias de los trabajadores y de su grado de organización sindical y política. Por otra parte, junto a la descualificación hay que sefialar también la recualificación y la sobrecualificación de otros trabajadores, especialmente de aquellos que trabajan en el diseño, reparación y mantenimiento de la alta tecnología ${ }^{11}$.

Sin embargo, para el grueso de la fuerza de trabajo, la tendencia general es a la descualificación, tanto del trabajo manual como intelectual y en este sentido no sólo habrá que aceptar que no hay un ajuste entre el sistema educativo y el sistema productivo, sino que ambos funcionan con dos lógicas diferencias.

\section{Hacia un nuevo realismo pedagógico}

Como en tantas otras esferas de la vida social, la crisis puso en evidencia la labilidad de las explicaciones teóricas con que nos manejábamos en la década del 60. La educación no puede todo ni es la llave maestra de la reforma social porque no puede tener otras características que las que le otorga el desarrollo histórico de cada sociedad.

Con la crisis no sólo se diluyó el narcisismo pedagógico sino que se desarrolló otra mirada sociológica sobre el fenómeno educativo, más introspectiva y crítica del propio

(8) EEC. Analysis and Projection of Regional Documentation on Regional Policy in the Community. Brusels, 1984, Pp. 54-58. El País, 16-XII-86.

(9) BRAVERMAN, HARRY, Labor and monopoly capital. Monthly Review Press, 1974, Edición en castellano, Editorial Era, México.

(10) LAWN, M., OZGA, J.: Techers, professionalism and class. The Falmer Press, Apple, M.: "Curricular form and the logic of technical control: building the possessive individual, en Apple, M. (edit) Cultural and Economic Reproduction in Education. Roulledge and Kegan Paul, 1982. Buswell, J. Social change and pedagogic change. British Jorunal of Sociology of Education Vol. 2 n.? 3, 1980.

(11) BURAWOY, M., The politics of production. Verso. Londres, 1985. 
quehacer. Diversas corrientes que van desde el Neo-Marxismo al Neo-Werianismo, de la Pedagogía Institucional y los análisis foucaultianos, nos pusieron en evidencia que la educación es poder y muchas veces un poder arbitrario que legitima otros poderes más arbitrarios aún.

La mirada crítica se extendió desde el papel de la educación en la división del trabajo y la ideología hasta los aspectos aparentemente más irrelevantes de la vida cotidiana en la escuela donde podemos reconocer manifestaciones de dominio, muchas veces no asumidas concientemente por los propios sujetos.

En otros trabajos he criticado las limitaciones de estos nuevos enfoques, en particular aquellos que ven en la educación exclusivamente un instrumento de reproducción al servicio de la clase dominante ${ }^{12}$. Sin embargo, pese a su unilateralidad, es preciso reconocer que contribuyeron a desmitificar la inocencia de la escuela y a contribuir a que hoy seamos más concientes de que la educación no es precisamente una práctica ingenua.

Pero es necesario avanzar más allá de la crítica y crear la posibilidad teórica de un espacio de cambio y transformación dentro de las propias instituciones educativas. No es una cuestión de voluntarismo, sino que casi diría, de exigencias de la realidad. Hoy sabemos que la educación no es per-se un agente de desarrollo y movilidad, que la socialización escolar no garantiza la aceptación del orden social, que el conocimiento escolar o académico tendrá una mínima aplicación en la vida laboral para la gran mayoría ... pero ello no significa que el mundo se venga abajo o que la educación haya perdido sentido.

La crisis abre precisamente las puertas a una nueva reflexión más realista. En principio, si la relación entre la educación y el trabajo es cada vez más incierta, la prepara. ción específica tiene poco sentido. Las famosas redes clasistas, que pre-anuncian la división del trabajo, como por ejemplo la F.P. y el B.U.P. no sólo aparecen como injustas sino como inútiles desde el punto de vista estrictamente técnico de los futuros empleos.

La adecuación al cambio técnico hoy no parece tener otra salida que una educación general, dúctil, polivalente, que recoja lo mejor de la tradición de lucha del movimiento obrero y la mejor tradición del pensamiento humanista. Lo que hace apenas 25 años aparecía como educación ornamental, sin la menor vinculación con el desarrollo y la movilidad, en suma, un despilfarro social, se empieza esbozar como una auténtica preparación para el cambio. Sólo una persona que tenga una buena formación básica en las ciencias puras puede tener instrumentos teóricos para enfrentar los procedimientos tecnológicos que cambian aceleradamente.

El cambio tecnológico es una dimensión a tener en cuenta para el retorno a los "principios", una dimensión necesaria pero no suficiente. La ductilidad y el ajuste a nuevas situaciones requiere también una nueva formación humanista, que no socialice en la apatía o.el conformismo. Son necesarios hombres y mujeres con espíritu crítico, que rechacen los dogmas de cualquier cuño, porque éstos además de conducir al oscurantismo como en el pasado, serán totalmente ineficaces en el futuro.

(12) MORGENSTERN DE FINKEL, S., Hegemonía y Educación. Revisla de Ciencias de la Educación, n. ${ }^{R} 13$ 14. Buenos Aires, 1974, "Transición Política y práctica educativa", próxima publicación, M.E.C. 